\title{
El uso de las TICs en la comunicación interna de la universidad: el caso de la Universidad Europea Miguel de Cervantes
}

\section{The Use of ICTs in Internal Communication of the University: The Case of Universidad Europea Miguel de Cervantes}

\author{
Alejandro Tapia Frade. Universidad Europea Miguel de Cervantes \\ José María Herranz de la Casa. Universidad Europea Miguel de Cervantes \\ Javier García González. Licenciado en Publicidad y Relaciones Públicas \\ Recibido: 31-III-2009 - Aceptado: 25-IX-2009 \\ Resumen:
}

El estudio de caso que se presenta tiene por objetivo el análisis del uso y la opinión de los directivos, profesores y alumnos de la Universidad Europea Miguel de Cervantes sobre las herramientas tecnológicas existentes. Mediante la encuesta se pone de manifiesto los altos niveles de conocimiento en las dos herramientas evaluadas: campus virtual y webmail (con un bajo nivel de uso efectivo, especialmente en el colectivo de estudiantes). En conclusión, si bien las TICs son positivamente valoradas, su uso efectivo presenta ciertas deficiencias que podrían subsanarse si se mejora el contenido de estos espacios y son usadas como canales esenciales de información entre los públicos implicados.

Palabras clave:

Comunicación interna, TICs en universidades, públicos internos, página web, campus virtual, webmail, diseño, uso de TICs.

\section{Abstract:}

The case study presented aims to use analysis and the opinion of management, professors and students of the Universidad Europea Miguel de Cervantes on existing technological tools. Through the survey it shows high levels of knowledge assessed in the two tools: a virtual campus and webmail (with a low level of use, especially in the student group). In conclusion, while ICTs are positively valued, their actual use has certain shortcomings which could be remedied by improving the content of these spaces are used as key channels of information between the public involved.

KeyWords:

Internal Communications, University ICTs, Internal Public, Websites, Virtual Campus, Webmail, ICTs Design, Use of ICTs. 


\section{Introducción}

La comunicación interna es una herramienta fundamental en la gestión empresarial, pero ha sido una cuestión especialmente poco tratada en el pasado, más aún en el ámbito universitario. Sin embargo, esta realidad está cambiando al irse incorporando en cada vez mayor medida departamentos de comunicación en estas organizaciones (Losada, 2004: 478).

La peculiaridad de la universidad como institución reside en que respecto de su "cliente" principal, el estudiante, no existe unanimidad en su consideración como público interno o externo. En ese sentido, y sea como fuere dicha categorización, es evidente que el colectivo de estudiantes es clave para conceptualizar la comunicación interna en las universidades (Palencia-Lefler, 2008: 278).

Es creciente el uso de las nuevas tecnologías de información en el ámbito de la comunicación interna. Además, los estudiantes universitarios están ampliamente familiarizados con el uso de estas tecnologías, como muestran los datos de algunos estudios (AIMC, EGM, $2^{\text {a }}$ Oleada, abril-junio 2009), por lo que habría que considerar que dichas herramientas son potencialmente útiles en el proceso comunicativo considerado en este artículo.

Todavía más, las universidades deben considerar el uso sistemático de las TICs como parte estratégica esencial de sus procesos comunicativos. Por ello, dichas tecnologías deben ser asumidas de forma paulatina ${ }^{1}$ (Gloria y Carbajo, 2005: 214).

La Universidad Europea Miguel de Cervantes cuenta con un destacado uso de TICs como parte esencial de la emisión y recepción de información entre sus públicos internos, por lo que se considera un sujeto de estudio adecuado al análisis que se pretende.

En concreto, el objetivo esencial de esta investigación es el análisis del uso y la opinión sobre herramientas tecnológicas en la comunicación interna en la UEMC. Por esta razón, podemos definir los siguientes objetivos empíricos de investigación:

- Analizar el uso y opinión sobre la herramienta tecnológica de la página web de la universidad, tanto desde el punto de vista estructural como de contenido.

1 Según Gloria y Carbajo (2005), "Las nuevas tecnologías implican nuevos retos que se deben ir asumiendo en la cotidianeidad, teniendo presente además la composición del público, en la medida que avancen los días, los nuevos integrantes del público serán -cada vez más- lectores virtuales para quienes la hoja de papel no será el soporte que da estructura y peso a la noticia impresa" 
Analizar el uso y opinión sobre la herramienta del campus virtual² como plataforma de gestión y comunicación en la UEMC.

Analizar el uso y opinión sobre el webmail de la universidad como herramienta de comunicación entre los distintos públicos de dicha universidad.

En definitiva, se pretende conocer el uso y opinión que los distintos públicos de esta universidad manifiestan en relación con las herramientas tecnológicas disponibles.

En lo relativo a los estudiantes universitarios, Chen y Peng (2008) señalaron diferencias significativas entre los que consumían internet de forma excesiva y los que hacían un uso moderado. De hecho, los consumidores moderados mostraron mejores relaciones con el personal de la universidad, obtuvieron mejores notas y mayor satisfacción en el aprendizaje.

Otro estudio a tomar en consideración es el de Martínez (2002), que trató de analizar las necesidades de recursos y de formación de los estudiantes en el empleo de las TICs como apoyo a su proceso de aprendizaje a partir de las encuestas realizadas a 289 alumnos de la Universidad de Oviedo. Este estudio concluyó que aunque la tasa de conexión a internet en los domicilios de estas familias era baja, y además los estudiantes señalaban insuficiente la conectividad ofrecida en dicha Universidad, éstos se mostraban seguros en su capacidad de uso de elementos como chats, buscadores, etc.

También hay que destacar la investigación de Azcorra Saloña (2001) sobre el uso de internet en la universidad española, en el que señala que el uso predominante en dicho ámbito corresponde a los navegadores web. La tesis doctoral de Villamizar Carrillo (2007) revela que los profesores universitarios no se consideran preparados en el ámbito de las TICs (Villamizar, 2007: 139). Y en esta línea también es interesante otro estudio realizado por Baigorri (2002) sobre una muestra de estudiantes universitarios en Badajoz. Finalmente, hay que reseñar otro estudio sobre estudiantes y profesores universitarios en Estados Unidos, que puso de manifiesto que la actitud de ambos hacia la web eran positivas, ya que ambos grupos la consideraban una herramienta válida en el proceso educativo (Jones y Jones, 2005: 125-135).

En cuanto a las hipótesis que pretende validar este trabajo, la principal es la siguiente: Los públicos internos de la UEMC conocen, valoran positivamente y usan habitualmente las herramientas tecnológicas de comunicación puestas a su disposición por la universidad en su relación con el resto de la comunidad uni-

2 El campus virtual recoge una serie de aplicaciones como registrar asistencia, colgar bibliografia, programas y otras informaciones acerca de la asignatura, calificar, cerrar actas, registrar el contenido de tutorías académicas y personales, ver fotografías de los alumnos, diversas consultas como calendario de exámenes, horarios de las asignaturas, consulta de calificaciones, etc. 
versitaria. Con el fin de poder corroborar la hipótesis citada, la investigación presenta las siguientes hipótesis secundarias:

1. El campus virtual y webmail son instrumentos de comunicación conocidos por parte de los públicos de la UEMC.

2. La página web y sus aplicaciones campus virtual y webmail son herramientas de comunicación usadas habitualmente por el PDI y los estudiantes.

3. La página web y su aplicación campus virtual son valorados positivamente por los públicos de la universidad.

\section{Metodología}

Este trabajo se presenta como un estudio de caso de una comunidad universitaria específica, la Universidad Europea Miguel de Cervantes, de titularidad privada y una antigüedad de 7 años (2002-2009). Por tanto, se excluye la generalización de resultados a toda la comunidad universitaria española.

El método de obtención de información fue la encuesta estructurada. En dicha encuesta se utilizaron esencialmente preguntas dicotómicas y basadas en escalas. Se determinó el uso de escalas pares, sin punto medio, con objeto de evitar valores centrales y facilitar la dispersión de valores al forzar la decisión en un sentido u otro. Este tipo de escalas han sido usadas en otros análisis de análoga temática (Marzal y Calzada, 2003: 57) y también defendidas por otros autores (Cox, 1980: 407 y Cafe, Santos, y Macedo, 2001: 73).

En cuanto a la técnica de muestreo, se utilizó el muestreo por cuotas. Se trataba de asegurar la adecuada representatividad del universo, por lo que se reprodujo en dicho muestreo la estructura exacta del universo de estudio en razón de sexo, posición en la universidad (PDI - PAS - Estudiantes), y dentro de estos últimos, los estudios cursados. El muestreo por cuotas constituye una síntesis de diferentes aspectos del muestreo por conveniencia, del muestreo según el criterio y del muestreo estratificado, y por ser intencionado, en todo caso ha de considerarse no probabilístico.

La idea que sostiene este procedimiento es que si la muestra representa correctamente las características poblacionales, ésta estará correctamente representada y será valida.

Como fácilmente puede comprenderse, esta idea es sólo parcialmente correcta. En efecto, sólo podemos "cuotar" las características más importantes y tenemos que dejar abierta la inclusión de otras muchas que también se relacionan con la variable de estudio. No hay que olvidar que en el interior de las cuotas 
el entrevistador probablemente actuará conforme a la ley del mínimo esfuerzo. Así, se sabe que hay ciertos tipos de personas excesivamente representados en este tipo de estudios, por ejemplo los porteros que son más fáciles de encontrar en un edificio. Lo mismo ocurre, por ejemplo, cuando se encuesta en bloques de pisos, los pisos más bajos suelen estar más representados que los más altos (Sánchez Guzmán, 1995: 278).

El procedimiento de investigación se ha desarrollado durante el curso lectivo 2007-2008 y la realización de la encuesta se realizó en el mes de abril de 2008.

El cuestionario contiene 69 preguntas, abiertas y cerradas, referentes a cuestiones de conocimiento en cuanto existencia, frecuencia, uso, preferencia y opinión, teniendo en cuenta las diferencias sociológicas de los 3 colectivos estudiados. Así, el cuestionario está subdividido en 3 cuestionarios -PDI (26 preguntas), PAS (17 preguntas) y Estudiantes (26 preguntas)-.

De las 134 encuestas válidas para el estudio, la mayor parte corresponde a estudiantes (100), a PDI (29) y PAS (5) debido a la proporcionalidad del universo de estudio. El universo de estudio constaba de 1.702 elementos, y estaba a su vez compuesto por 1.557 estudiantes, 125 PDI y 20 PAS. Para la explotación de datos se utilizó el software específico SPSS v. 14.

\section{Resultados}

A continuación se exponen los resultados relevantes obtenidos que dan respuesta a los objetivos de investigación. Se han dividido en tres subapartados que responden al conocimiento, a la frecuencia de uso y finalmente a la valoración, tanto de forma como de contenido, de las herramientas planteadas.

\subsection{Conocimiento de las TICs en la UEMC}

Respecto al conocimiento de las herramientas que la universidad objeto de estudio pone a disposición de los tres públicos estudiados (PDI, PAS y Estudiantes), se trataba de evaluar si las dos herramientas de comunicación analizadas (campus virtual y webmail) tenían la suficiente divulgación, o por el contrario, si no la tenían, sería necesaria una correcta campaña de divulgación de las mismas.

El campus virtual es una herramienta conocida por casi toda esta comunidad universitaria. Así, el 96\% del colectivo Estudiantes y el 100\% del colectivo PDI (el PAS no dispone de esta herramienta de comunicación) afirman conocer el campus virtual. 
Otra cuestión es el webmail, ampliamente conocido por PDI $(93,1 \%)$ y PAS (100\%), pero no tan conocido por el colectivo Estudiantes, ya que apenas el 56,6\% del mismo afirma conocerla. Es relevante este dato porque en apenas 4 meses (enero de 2008) de su puesta en funcionamiento para los estudiantes, sólo la mitad conocen su existencia, mientras que PDI y PAS la tienen desde el nacimiento de la universidad (2002). Sería por tanto interesante cierta labor divulgativa hacia el colectivo Estudiantes.

\subsection{Frecuencia de uso de las TICs en la universidad}

Los datos presentados en este epígrafe parten de la consideración siguiente: para poder usar una herramienta es condición sine qua non conocerla antes. Por ello, los porcentajes se refieren a aquellos que declararon conocer cada una de las herramientas tratadas.

Respecto a la frecuencia de uso de la página web, el colectivo más aplicado es el del PAS, que afirma en su totalidad usar la página web todos los días, si bien el PDI también la usa frecuentemente, aunque no con esa intensidad. Estos datos pueden verse afectados porque no todo el PDI tiene dedicación exclusiva, y por tanto no ve necesario tal nivel de uso.

Los datos del colectivo Estudiantes muestran una dispersión mucho mayor, si bien el 40,4\% la visita al menos una vez a la semana. No obstante, en dicha página web se informa puntualmente de las novedades académicas y posibles noticias de interés para este colectivo, por lo que cabe sugerir que usan otras formas de conocimiento para informarse o que simplemente el contenido alojado en este sitio web no se ajusta a sus demandas. Los datos pueden verse en la tabla I.

Respecto a la frecuencia de uso de la herramienta campus virtual, al igual que en el caso de la página web, los datos difieren notablemente considerando los diferentes públicos. Así, el colectivo PDI afirma mayoritariamente usar el campus virtual todos los días (72,4\%), y en menor medida, dos veces por semana (20,7\%). Hay que considerar en este caso dos hechos relevantes:

a) El campus virtual es una herramienta de gestión académica esencial para el PDI, ya que desde ella se gestiona la información operativa diaria.

b) Como ya se mencionó anteriormente, no todo el colectivo PDI tiene régimen de exclusividad, por lo que existe una parte de este colectivo que no necesita tal intensidad de uso.

Por tanto, a priori debería constituir un elemento de uso diario para la parte de PDI que tiene régimen de exclusividad, ya que en esta universidad, de titularidad privada, si se tiene tal régimen de contratación, el PDI tiene obligación laboral de asistencia al campus todos los días. 
El colectivo Estudiantes muestra una frecuencia de uso notablemente inferior, como puede apreciarse en la tabla II. Esto es debido a que la información que se puede encontrar en esta herramienta está mucho más limitada y atemporal. Los alumnos encuentran en este espacio información sobre horarios, exámenes y calificaciones.

Los datos referentes a la frecuencia de uso del webmail por parte de los distintos colectivos de la universidad muestran una realidad bien distinta, como puede apreciarse en la tabla III.

Si bien algo más de la mitad del colectivo PAS y la amplia mayoría del PDI afirma usarlo todos los días, dicha frecuencia de uso únicamente se da en una parte marginal del colectivo Estudiantes, que muestra periodicidades de uso muy superiores. Además, existe un dato ampliamente relevante: el 51,8\% del total de dicho colectivo afirma conocerlo pero no utilizarlo. Esta circunstancia podría modificarse si el webmail fuera el instrumento preferente de comunicación entre profesores y alumnos, por lo que debe considerarse esta posibilidad.

\subsection{Valoración de las TICs en la universidad}

Una vez expuestas las cuestiones de conocimiento y uso de las TICs en la universidad, abordamos ahora la valoración que dichas herramientas soportan por parte de los tres colectivos. Se ha estructurado la valoración de las TICs en dos sentidos 3 : de un lado, el aspecto que abarca cuestiones de forma como el diseño, y de otro, el contenido que las herramientas tienen alojado y su pertinencia.

\subsubsection{Valoración del aspecto de las TICs en la universidad}

La premisa con que se parte en este caso es la siguiente: condición sine qua non para valorar una herramienta es conocerla. No se consideró que además de conocerla tuvieran que usarla con frecuencia, pues cabe la posibilidad de valorar el desempeño de una herramienta aunque no se use habitualmente.

\footnotetext{
3 Tomamos en consideración el modelo de análisis de Zang y Dran (2000) y la posterior aportación de Hassan Montero (2006) que basan el análisis de la satisfacción web alrededor del binomio satisfacción - frustración. En dicho modelo se ofrecen factores higiénicos (orientados a la no frustración), que son la accesibilidad y la findability -considerada como la la medida de la capacidad del usuario para encontrar la información buscada en un tiempo razonable-, y factores motivadores, orientados a la satisfacción, que son la utilidad, la estética y la credibilidad. La usabilidad se describe como factor orientado simultáneamente a la satisfacción y a la no frustración, siendo por tanto un factor estructural de primer orden en el modelo. En el caso de sitios web de entretenimiento, la estética representa en factor más importante en el modelo, y por el contrario el factor denominado credibilidad tiene poca presencia, aunque dicho factor tiene importancia en sitios web que no tienen por objeto el entretenimiento. Los factores utilidad y findability resultaron ser los más intensos independientemente del dominio web estudiado. Algo menos importantes, también independientemente del diseño web, fueron los factores usabilidad y accesibilidad. La conclusión fundamental del análisis de Zang y Dran es que los factores higiénicos resultan de mayor prioridad debido a su condición de prerrequisito para los factores motivadores, mientras que estos últimos ofrecen valor añadido.
} 
Así, para valorar la página web y el campus virtual se usó una escala de seis posiciones (de cero a cinco), sin punto medio, para evitar tendencias de valoración hacia el centro y forzar la decisión en algún sentido. En ambas herramientas se midió el diseño globalmente considerado, y varios elementos que en el primero inciden, como son: los colores usados y su relación con lo corporativo; la rapidez de carga; la facilidad de utilización y la disposición y claridad de los menús. Más allá de la mera puntuación, también se trataba de establecer la importancia de cada uno de ellos en la consideración del diseño global en ambas aplicaciones, para lo que se recurrió a un análisis de regresión lineal múltiple.

Al observar los valores medios arrojados hacia la consideración global del diseño -descritos en tabla IV-, observamos una valoración positiva en los tres colectivos. Además, el uso de colores corporativos es un hecho altamente percibido en los tres colectivos.

La rapidez de carga es el elemento con menor puntuación, si bien hay que señalar que dicha rapidez no sólo tiene que ver con la página, sino también con elementos que lo condicionan y son ajenos a la misma -por ejemplo, el funcionamiento de la red local o del ancho de banda de que se disponga cuando la conexión se realiza desde el exterior de la universidad -. La facilidad de uso de la página web registra valores dispares según el colectivo que lo cuantifique ${ }^{4}$.

Finalmente, el último elemento a valorar es la claridad y disposición de los menús. Al igual que el caso anterior, la disparidad de valoraciones según el colectivo es notoria, aunque mantiene el mismo sentido.

Respecto de la importancia que estos elementos tienen, los tres colectivos opinan que el diseño es acertado como consideración global. Se observa que en un análisis preliminar, las correlaciones parciales entre el buen diseño y el resto de variables son siempre en sentido positivo, pero no especialmente brillantes (todas entre 0,3 y 0,6), lo que sugiere cierta debilidad de asociación entre las mismas. El análisis de regresión lineal múltiple posteriormente ejecutado señala que podrían existir otros factores explicativos no contemplados en la calificación otorgada al diseño (el coeficiente R cuadrado es 0,42 ). Los detalles pueden verse en las tablas Vy VI.

Respecto a la puntuación otorgada por término medio por los dos colectivos en el caso de la herramienta campus virtual (recordamos que el colectivo PAS no dispone de esta herramienta), hay que señalar que la valoración de los distintos colectivos respecto a la consideración del color y su proximidad al corporativo

4 Liu, Liao y Pratt (2009) determinaron en un estudio que la utilidad percibida de un contexto de aprendizaje a través de web no se correlacionaba positivamente con el aumento de recursos de audio y vídeo en dicha web. Sin embargo, sí hallaron correlaciones positivas entre dicho aumento de recursos y niveles más altos de concentración. 
ofrecen gran similitud, pero la dispersión de criterios es muy superior en el caso del PDI, tal y como puede apreciarse en la tabla VII.

La rapidez de carga es el siguiente elemento a considerar, y tal y como ocurre con la página web, la valoración del colectivo Estudiantes es inferior a la del PDI. Al igual que en el caso anterior, existe mayor dispersión de datos en el caso del colectivo PDI. La facilidad de uso de la herramienta arroja los valores por término medio más altos, muy similares en ambos colectivos.

Al igual que en el caso anterior, se intentó conocer el peso que las mencionadas variables tenían en la consideración de la variable diseño. Las correlaciones bivariadas entre el buen diseño y el resto de variables teóricamente explicativas arrojan valores en sentido positivo bastante más altas que en el caso anterior (todas entre 0,54 y 0,65). El análisis de regresión múltiple posteriormente ejecutado señala que el porcentaje explicado se mantiene respecto al modelo anterior (R cuadrado tiene valor de 0,43), y también en este caso existen otros factores no contemplados que en principio deberían ayudar a explicar la consideración de un buen diseño. Los resultados del modelo pueden apreciarse en las tablas VIII y IX.

Asimismo, se consideró cuál de los dos diseños, el de la página web y el del campus virtual, es más apreciado. Los datos muestran que en general gusta más el diseño de la página web (52,4\% sin grandes variaciones según el colectivo) que el del campus virtual (25\% en ambos casos), si bien hay un importante porcentaje de indecisos (22,6\% sin variaciones notorias en ambos colectivos). Se da la circunstancia de que el diseño de la nueva página web data de noviembre 2007, mientras que el diseño del campus virtual lleva inalterado desde los inicios (siete años), lo que sugiere que el nuevo diseño es en mayor medida del agrado de los colectivos estudiados en este caso.

\subsubsection{Valoración de los contenidos de las TICs en la universidad}

Una vez revisada la valoración del diseño de las herramientas tecnológicas en la universidad, pretendemos ahora conocer si el contenido de éstas es el adecuado, y además qué elementos son superfluos y cuáles no son incluidos. Las preguntas realizadas en el cuestionario eran abiertas para el caso de la página web y el campus virtual, realizándose posteriormente la categorización de las mismas, por lo que se presentan únicamente las que mostraban mayor coincidencia de opinión.

Respecto a la página web, el 72,4\% del colectivo PDI añadiría más contenidos a la página web, concretamente información con los cambios de horario, fechas de exámenes y prácticas (14,3\%), mejoras en la navegación (9,5\%), foros para consultas de datos (9,5\%), premios, becas y convocatorias (9,5\%), la historia de la universidad $(9,5 \%)$ y una guía universitaria on-line $(9,5 \%)$. 
El colectivo Estudiantes muestra mayor indiferencia a esta cuestión, ya que únicamente el 54,5\% de los estudiantes añadiría elementos a la página web, en concreto menús más claros (20,4\%), foros para consulta de datos (18,5\%), galerías de imágenes con las actividades y ceremonias realizadas $(7,4 \%)$ y mejoras en la agenda y el calendario $(7,4 \%)$.

También se cuestionó si había algún elemento que los colectivos desearan eliminar de la página web. Respecto a esta cuestión, la indiferencia es mucho mayor: tan sólo 6,9\% del PDI y el 14,1\% del colectivo Estudiantes quitaría algún elemento de la página web. El PDI quitaría actividades ajenas a la comunidad universitaria y restringiría el acceso a contenidos a colectivos ajenos a la universidad. El colectivo Estudiantes, además de los dos elementos ya mencionados -el último de ellos en menor medida-, quitarían las fotografías que aparecen en la parte superior de la página web.

Respecto al campus virtual parece haber mayor sintonía en los contenidos que en la página web, ya que el 48,3\% del PDI y el 39,6\% del colectivo Estudiantes añadiría elementos, y tan solo el 3,4\% del PDI y el 4,2\% del Estudiantes quitaría elementos. Concretamente el PDI añadiría un calendario personalizado (28,6\%), cursos para cada titulación (14,3\%) y la posibilidad de enviar o recibir trabajos o prácticas $(7,1 \%)$. El colectivo Estudiantes añadiría el porcentaje de ausencias a clase $(29,7 \%)$, un calendario personalizado $(8,1 \%)$ y en menor medida cursos por titulaciones $(5,4 \%)$ y la posibilidad de enviar trabajos y prácticas $(5,4 \%)$. Tal y como se ha mencionado anteriormente, tan sólo un pequeño porcentaje de PDI y Estudiantes eliminaría elementos del campus virtual. De este pequeño porcentaje, únicamente es notorio en el colectivo Estudiantes la ampliación del periodo en que están visibles las calificaciones.

Con referencia al webmail, los colectivos PDI y PAS afirman utilizarlo como herramienta tanto de comunicación con otros estamentos de la universidad - 96,3\% del PDI y 80\% del PAS- (PDI con PAS, PDI y PAS con decanatos, vicerrectorados, etc.), y todavía más, en su relación con otros compañeros de similar posición en la universidad (92,3\% del PDI y la totalidad del PAS). El colectivo Estudiantes que usa el webmail afirma hacerlo mayoritariamente para realizar gestiones con la universidad $(66,7 \%)$ y en mucha menor medida en su relación con compañeros (18,5\%).

En el momento de realizar la encuesta, el gabinete de comunicación enviaba con periodicidad semanal una agenda con la información relevante para la comunidad universitaria, pero únicamente a los colectivos PDI y PAS. Preguntado al tercer colectivo acerca de la posibilidad de este servicio, el 71,4\% afirmaba que le gustaría recibir esta información -desde octubre 2008 los alumnos reciben una agenda semanal con toda la información de interés que elabora el gabinete de comunicación en colaboración con todos los servicios de la UEMC-, que además pudiera servir como elemento de estímulo para el uso del mismo. Todavía más, se 
podría incentivar el uso de esta herramienta entre el colectivo Estudiantes si el resto de colectivos lo usara de forma esencial en su relación con éste.

Finalmente, la totalidad del PAS, el 81,5\% del PDI y el 87,5\% de los estudiantes consideraría positivo el uso del webmail para el envío de sugerencias, comentarios y opiniones. Para ello bastaría con habilitar una cuenta de correo e implementar los procesos de gestión necesarios para su funcionamiento.

\section{Conclusiones}

Con el fin de validar la hipótesis central de este trabajo, recurrimos en primer lugar al contraste de las tres hipótesis secundarias.

Respecto a la primera hipótesis secundaria, "El campus virtual y webmail son instrumentos de comunicación conocidos por parte de los públicos de la UEMC”, los datos señalan el amplio conocimiento del campus virtual en los dos colectivos estudiados (el PAS, tal y como se mencionó anteriormente, no dispone de esta herramienta), por lo que se considera afirmativa la respuesta.

Sin embargo, los datos muestran una realidad bien diferente para el caso de la herramienta webmail. Si bien los datos muestran un alto nivel de conocimiento para los colectivos de PAS y PDI, los resultados para el colectivo Estudiantes no son buenos, ya que apenas el 56,6\% afirman conocerla. Es por tanto necesaria la realización de una labor divulgativa destinada al conocimiento de la herramienta. Por lo tanto, no podemos concluir que exista conocimiento de esta herramienta por parte de los estudiantes.

En relación con la segunda hipótesis secundaria, "La página web y sus aplicaciones campus virtual y webmail son herramientas de comunicación usadas habitualmente el PDI y los estudiantes”, queda claramente lastrada por el bajo conocimiento mostrado por el colectivo Estudiantes de la herramienta webmail, ya que no se puede usar frecuentemente aquello que no se conoce. En concreto, los datos de frecuencia de uso respecto a la página web muestran alto nivel de frecuencia en el caso del PAS y PDI, y más bajo en el caso de Estudiantes, pero en cualquier caso la frecuencia de uso de este colectivo es suficientemente relevante como para aceptar esta parte de la hipótesis. Los datos mostrados para frecuencia de uso de la herramienta campus virtual acusan la misma tendencia anteriormente mostrada: el colectivo PDI muestran gran frecuencia de uso y los datos para el colectivo Estudiantes son inferiores, pero que estimamos suficientes (casi el 65\% afirma usar dicha herramienta todos los meses).

Respecto a la tercera herramienta, conviene considerar, más allá de los datos de frecuencia de uso de que disponemos (que no estimamos suficientes para validar la premisa), que difícilmente puede usarse algo 
que no se conoce. Así, además de la labor divulgativa antes planteada, sería interesante considerar el uso de este canal de información como exclusivo en la relación del colectivo Estudiantes con los otros públicos de la universidad, forzando así al mencionado colectivo en su uso y promoción.

En referencia a la tercera hipótesis secundaria, "La página web y su aplicación campus virtual son valorados positivamente por los públicos de la universidad”, los datos permiten validar la hipótesis planteada para las dos herramientas.

Los datos para la página web muestran valoración positiva en aspecto y parcialmente adecuada en contenidos, ya que por una parte no se eliminaría lo existente, pero se añadirían algunos elementos, especialmente en el caso del colectivo PDI.

Los datos de la herramienta campus virtual permiten también señalar valoración positiva en lo que atañe al aspecto, y mayor adecuación de contenido a las expectativas de los dos colectivos objeto de estudio que en el caso de la página web.

Finalmente, respecto de la hipótesis central planteada en este trabajo, "Los públicos internos de la UEMC conocen, valoran positivamente y usan habitualmente las herramientas tecnológicas de comunicación puestas a su disposición por la universidad en su relación con el resto de la comunidad universitaria”, los datos, según se ha explicado anteriormente, no permiten validar dicha hipótesis, ya que es condición para tal hecho que todas las hipótesis parciales se vieran cumplidas, hecho que no sucede con las dos primeras, debido esencialmente al bajo conocimiento (y consecuentemente baja frecuencia de uso) de un colectivo (Estudiantes) para una de las herramientas (webmail), pero considerando positivo tanto el conocimiento (y la valoración y frecuencia de uso) para los tres colectivos en el caso del resto de herramientas estudiadas.

\section{Discusión final}

Las TICs pueden sin duda contribuir a mejorar sensiblemente tanto los procesos de gestión operativa diaria de la universidad como la relación entre los distintos públicos que en ella confluyen. El caso descrito pone de manifiesto un hecho cuando menos interesante: se dispone de recursos tecnológicos pero no se hace en líneas generales un uso efectivo de ellos. La cuestión es por qué. De un lado, ha quedado de manifiesto que el contenido del campus virtual y la página web no se ajusta a las demandas requeridas, por lo que habría que analizar cuáles son los items de información en mayor medida requeridos y estructurarlos adecuadamente en la página web y el campus virtual. Ahora bien, la heterogeneidad de públicos dificulta notablemente este hecho, que sin duda debería ser objeto de una investigación explícita. 
Por otra parte, también se ha señalado que el colectivo de los estudiantes no usa la cuenta de correo con la frecuencia deseada, que podría, como ya se ha mencionado, incrementarse si se usara como canal de comunicación imperativa entre estudiantes y personal docente. Sin embargo, debería monitorizarse este proceso, ya que el presumible incremento de uso debe llevar aparejada la adaptación de recursos destinados a tal efecto, en especial el tamaño de buzón y la eficiencia de las conexiones.

Además, la mejora de estos elementos podría agilizar la relación entre los distintos públicos, e incluso suponer factores de motivación y rendimiento importantes en dichos colectivos, que podría redundar en un incremento de eficiencia de este estrato educativo.

Finalmente hay que reseñar que los resultados obtenidos en este estudio han servido a la universidad y al departamento de comunicación para mejorar la comunicación con sus públicos internos -PDI, PAS y alumnos-. Cuando se habla de este tipo de públicos, entendemos que el uso de las nuevas tecnologías debe ir acompañado de herramientas y canales de comunicación directa y participativa -reuniones, grupos de discusión y mejora, etc.-. Así, la complementariedad de lo presencial y lo tecnológico ha resultado de gran eficacia en la gestión de la comunicación de la UEMC durante el proceso de puesta en marcha e implantación del Espacio Europeo de Educación Superior durante el año 2009.

\section{Tablas de datos}

Tabla I. Frecuencia de uso de la página web de la universidad (www.uemc.es) por colectivo.

\begin{tabular}{|c|c|c|c|c|}
\hline \multirow[t]{2}{*}{ Frecuencia de visita } & \multicolumn{3}{|c|}{ Público Interno } & \multirow[t]{2}{*}{ Total } \\
\hline & Estudiantes & PDI & PAS & \\
\hline Todos los días & $2,0 \%$ & $82,8 \%$ & $100,0 \%$ & $23,3 \%$ \\
\hline $\begin{array}{l}\text { Aproximadamente dos } \\
\text { veces por semana }\end{array}$ & $17,2 \%$ & $13,8 \%$ & & $15,8 \%$ \\
\hline Una vez por semana & $21,2 \%$ & & & $15,8 \%$ \\
\hline Una vez cada dos semanas & $16,2 \%$ & $3,4 \%$ & & $12,8 \%$ \\
\hline Una vez al mes & $28,3 \%$ & & & $21,1 \%$ \\
\hline Una vez al cuatrimestre & $8,1 \%$ & & & $6,0 \%$ \\
\hline Una vez al año & $1,0 \%$ & & & ,8\% \\
\hline No la utilizo & $5,1 \%$ & & & $3,8 \%$ \\
\hline $\mathrm{NS} / \mathrm{NC}$ & $1,0 \%$ & & & $8 \%$ \\
\hline Total & $100,0 \%$ & $100,0 \%$ & $100,0 \%$ & $100,0 \%$ \\
\hline
\end{tabular}

Fuente: elaboración propia 
Tabla II. Frecuencia de uso de la herramienta campus virtual por colectivo

\begin{tabular}{|lccc|}
\hline Frecuencia de visita & Estudiantes & $\begin{array}{c}\text { Público Interno } \\
\text { PDI }\end{array}$ & Total \\
\hline Todos los días & $1,1 \%$ & $72,4 \%$ & $17,7 \%$ \\
\hline $\begin{array}{l}\text { Aproximadamente dos } \\
\text { veces por semana }\end{array}$ & $8,4 \%$ & $20,7 \%$ & $11,3 \%$ \\
\hline Una vez por semana & $16,8 \%$ & $3,4 \%$ & $13,7 \%$ \\
\hline Una vez cada dos semanas & $12,6 \%$ & $3,4 \%$ & $10,5 \%$ \\
\hline Una vez al mes & $33,7 \%$ & & $25,8 \%$ \\
\hline Una vez al cuatrimestre & $6,3 \%$ & & $13,8 \%$ \\
\hline No la utilizo & $17,9 \%$ & & $2,4 \%$ \\
\hline $\begin{array}{l}\text { Solo en periodo de exámenes, } \\
\text { para consultar mis calificaciones }\end{array}$ & $3,2 \%$ & $100,0 \%$ \\
\hline Total & $100,0 \%$ & $100,0 \%$ & \\
\hline
\end{tabular}

Fuente: elaboración propia

Tabla III. Frecuencia de uso de la herramienta webmail por colectivo

\begin{tabular}{|lcccc|}
\hline Frecuencia de visita & \multicolumn{2}{c}{ Público Interno } & PAS & Total \\
\hline Todos los días & $3,6 \%$ & $81,5 \%$ & $60,0 \%$ & $30,7 \%$ \\
\hline $\begin{array}{l}\text { Aproximadamente dos } \\
\text { veces por semana }\end{array}$ & $8,9 \%$ & $7,4 \%$ & & $8,0 \%$ \\
\hline Una vez por semana & $12,5 \%$ & & $20,0 \%$ & $9,1 \%$ \\
\hline Una vez cada dos semanas & $7,1 \%$ & & $4,5 \%$ \\
\hline Una vez al mes & $8,9 \%$ & & $5,7 \%$ \\
\hline Una vez al cuatrimestre & $1,8 \%$ & $3,7 \%$ & $1,1 \%$ \\
\hline No lo utilizo & $51,8 \%$ & $7,4 \%$ & $35,2 \%$ \\
\hline NS/NC & $5,4 \%$ & $100,0 \%$ & $5,7 \%$ \\
\hline Total & $100,0 \%$ & $100,0 \%$ & $100,0 \%$ \\
\hline
\end{tabular}

Fuente: elaboración propia 
Tabla IV. Valoración media de elementos de la página web (www.uemc.es) por colectivo

\begin{tabular}{|c|c|c|c|c|c|c|}
\hline \multirow[t]{3}{*}{ ítem } & \multicolumn{6}{|c|}{ Público Interno } \\
\hline & \multicolumn{2}{|c|}{ Estudiantes } & \multicolumn{2}{|l|}{ PDI } & \multicolumn{2}{|l|}{ PAS } \\
\hline & $\begin{array}{l}\text { Media } \\
(0-5)\end{array}$ & $\begin{array}{l}\text { Desv. } \\
\text { Típica }\end{array}$ & $\begin{array}{l}\text { Media } \\
(0-5)\end{array}$ & $\begin{array}{l}\text { Desv. } \\
\text { Típica }\end{array}$ & $\begin{array}{l}\text { Media } \\
(0-5)\end{array}$ & $\begin{array}{l}\text { Desv. } \\
\text { Típica }\end{array}$ \\
\hline $\begin{array}{l}\text { Diseño globalmente } \\
\text { considerado }\end{array}$ & 2.73 & 1.08 & 2.72 & 1.09 & 3.80 & 0.83 \\
\hline Los colores son corporativos & 3.38 & 1.09 & 3.48 & 1.12 & 4.20 & 0.84 \\
\hline Carga con rapidez & 2.53 & 1.20 & 2.66 & 1.14 & 3.20 & 0.45 \\
\hline Es fácil de usar & 2.71 & 1.14 & 3.14 & 1.06 & 3.80 & 0.84 \\
\hline Los menús están claros & 2.48 & 1.17 & 2.79 & 1.42 & 3.40 & 0.89 \\
\hline
\end{tabular}

Fuente: elaboración propia

Tabla V. ANOVA de modelo de regresión múltiple para la página web, todos los colectivos

\begin{tabular}{|lccccc|}
\hline Modelo & Suma de cuadrados & gl & Media cuadrática & F & Sig. \\
\hline 1Regresión & 64,477 & 4 & 16,119 & 22,708 &, $000(a)$ \\
\hline Residual & 90,152 & 127 &, 710 & & \\
\hline Total & 154,629 & 131 & & \\
\hline $\begin{array}{l}\text { a) Variables predictoras: (Constante), Los menús están claros, Los colores son corporativos, Carga con ra- } \\
\text { pidez, Fácil utilización } \\
\text { b) Variable dependiente: Tiene un buen diseño }\end{array}$ & & & \\
\hline
\end{tabular}

Fuente: elaboración propia 
Tabla VI. Coeficientes en modelo de regresión múltiple para la página web, todos los colectivos

\begin{tabular}{|lccccc|}
\hline Modelo & \multicolumn{2}{l}{$\begin{array}{l}\text { Coeficientes no } \\
\text { estandarizados }\end{array}$} & \multicolumn{2}{c|}{$\begin{array}{l}\text { Coeficientes } \\
\text { estandarizados }\end{array}$} & Sig. \\
\hline & B & Error típ. & Beta & t &, 166 \\
\hline 1 Constante) &, 375 &, 269 & & 1,392 &, 000 \\
\hline Los colores son corporativos &, 448 &, 074 &, 453 & 6,027 &, 705 \\
\hline Carga con rapidez &, 027 &, 072 &, 030 &, 379 &, 215 \\
\hline Fácil utilización &, 121 &, 097 &, 126 & 1,246 &, 051 \\
\hline Los menús están claros &, 174 &, 089 &, 195 & 1,967 & \\
\hline
\end{tabular}

Fuente: elaboración propia

Tabla VII. Valoración media de elementos del campus virtual por colectivo

\begin{tabular}{|lcccc|}
\hline ítem & Público Interno & & \\
\hline & Estudiantes & & PDI & \\
\hline & Media (0-5) & Desv. Típica & Media (0-5) & Desv. Típica \\
\hline Diseño globalmente considerado & 2.70 & 1.31 & 2.59 & 1.21 \\
\hline Los colores son corporativos & 2.93 & 1.09 & 2.90 & 1.59 \\
\hline Carga con rapidez & 2.40 & 1.12 & 2.71 & 1.30 \\
\hline Es fácil de usar & 3.04 & 1.22 & 3.04 & 1.26 \\
\hline Los menús están claros & 2.85 & 1.29 & 3.07 & 1.36 \\
\hline
\end{tabular}

Fuente: elaboración propia

Tabla VIII. ANOVA de modelo de regresión múltiple para el campus virtual, todos los colectivos

\begin{tabular}{|lccccc|}
\hline Modelo & Suma de cuadrados & gl & Media cuadrática & F & Sig. \\
\hline 1 Regresión & 71,348 & 4 & 17,837 & 22,366 &, $000(\mathrm{a})$ \\
\hline Residual & 93,308 & 117 &, 798 & & \\
\hline Total & 164,656 & 121 & & & \\
\hline
\end{tabular}

Fuente: elaboración propia 
Tabla IX. Coeficientes en modelo de regresión múltiple para el campus virtual, todos los colectivos

\begin{tabular}{|lccccc|}
\hline Modelo & \multicolumn{2}{c}{$\begin{array}{c}\text { Coeficientes no } \\
\text { estandarizados }\end{array}$} & \multicolumn{2}{c|}{$\begin{array}{c}\text { Coeficientes } \\
\text { estandarizados }\end{array}$} & \\
\hline & $\mathrm{B}$ & Error típ. & Beta & $\mathrm{t}$ & Sig. \\
\hline 1 (Constante) &, 461 &, 249 & & 1,852 &, 067 \\
\hline Carga con rapidez &, 036 &, 089 &, 036 &, 403 &, 688 \\
\hline Fácil utilización &, 052 &, 117 &, 054 &, 440 &, 661 \\
\hline Los menús están claros &, 342 &, 104 &, 376 & 3,306 &, 001 \\
\hline Los colores son corporativos &, 314 &, 080 &, 326 & 3,916 &, 000 \\
\hline
\end{tabular}

Fuente: elaboración propia

\section{Referencias bibliográficas}

AIMC. Estudio General de Medios. (2009): “Audiencia de Internet, 2a Oleada, abril-junio 2009”. [Consultado el 1 de octubre de 2009]. Disponible en: http://www.aimc.es/

Azcorra Saloña, A. y Ortega Castro, V. (2001): "El uso de Internet en la Universidad Española” II Congreso Internacional de la Lengua, Valladolid, 16-19 Octubre. [Consultado el 1 de octubre de 2008]. Disponible en: http://cvc.cervantes.es/obref/ congresos/valladolid/ponencias/

Baigorri, A. y Fernández, R. (2002): "Universitarios de primera y universitarios de segunda. La fractura digital en la universidad”, en Infodex (coord.): Retos de la alfabetización tecnológica en un mundo en Red: Educación. Mérida: Consejería de Educación, Ciencia y Tecnología, pp. 50-60

Cafe, L., Santos, C. y Macedo, F. (2001): "Proposta de um método para escolha de software de automação de bibliotecas", Ci. Inf., May/Aug., vol. 30, no. 2, pp. 70-79.

Chen, Y. F. y Peng, S. S. (2008): "University students' Internet use and its relationships with academic performance, interpersonal relationships, psychosocial adjustment, and self-evaluation”, Cyberpsichology \& behavior, vol. 11 (4), pp. 467-469.

Cox, E. P. (1980): “The Optimal Number of Responses Alternatives for a Scale : A Review”, Journal of Marketing Research, vol. 17, pp. 407-422.

Gloria Donoso, C. y Carbajo Centeno, P. (2005): "Periódicos universitarios digitales o impresos. La responsabilidad de construir sentido”, en Marín Ruiz, A.; Trilles Rodríguez, I. y Zamarrón Garza, G. (coods.): Mass Media y Universidad. El reto de la comunicación en las universidades. Granada: Diálogo Iberoamericano, pp. 183-220. 
Hassan Montero, Y. (2006): “Factores del diseño web orientado a la satisfacción y no frustración de uso”, Revista Española de Documentación científica, vol. 29 (2), pp. 239-257.

Jones, G. H. y Jones, B. H. (2005): "A comparison of teacher and student attitudes concerning use and effectiveness of Webbased course management software”, Education Technology \& Society, vol. 8 (2), pp. 125-135.

Liu, S. H., Liao, H. L. y Pratt, J. A. (2009): “Impact of media richness and flow on e-learning technology acceptance”, Computers \& Education, vol. 52 (3), pp. 599-607

Losada Díaz, J. C. (2004): Gestión de la comunicación en las organizaciones. Barcelona: Ariel Comunicación.

Martínez González, R. A. et al. (2002): "El uso de las tecnologías de la información y la comunicación por parte de los estudiantes universitarios y su diferenciación en función del género", II Congreso europeo de tecnologías de la información en la educación y la ciudadanía: una visión crítica, Barcelona, 26-28 de junio. [Consultado el 30 de septiembre de 2008]. Disponible en: http://web.udg.es/ tiec/orals/c85.pdf.

Marzal García-Quismondo, M. A. y Calzada Prado, F. J. (2003): “Un análisis de necesidades y hábitos informativos de estudiantes universitarios en Internet”, Binaria: Revista de comunicación, cultura y tecnología, vol. 3, p. 57

Palencia-Lefler Ors, M. (2008): "La incomunicación interna en la Universidad española", Revista Latina de Comunicación Social, vol. 63, pp. 277-286. La Laguna (Tenerife): Universidad de La Laguna, recuperado el 27 de septiembre de 2008, de http://www.ull.es/publicaciones/latina/_2008/22_36_UPF/Manel_Palencia.html

Sánchez Guzmán, J. R. (1995): Marketing: conceptos básicos y consideraciones fundamentales. Madrid: McGraw Hill.

Villamizar Carrillo, L. P. (2007): Estrategias de formación de profesores universitarios para el uso de las tecnologías de información y comunicaciones (TICs) a partir de un sistema de aprendizaje Let me learn: dos estudios de caso. Tesis Doctoral, Universidad Rovira i Virgili (Tarragona).

Zhang, P. y Dran, G. M. (2000): “Satisfiers and Dissatisfiers: A Two-Factor Model for Website Design and Evaluation”, Journal of the American Society for Information Society, vol. 51 (14), pp. 1253-1268. 